Check for updates

Cite this: Phys. Chem. Chem. Phys., 2019, 21, 14350

Received 19th October 2018, Accepted 17th December 2018 DOI: $10.1039 / c 8 c p 06536 e$

rsc.li/pccp

\title{
The role of symmetric functionalisation on photoisomerisation of a UV commercial chemical filter $\dagger$
}

\author{
Jack M. Woolley, ${ }^{a}$ Jack S. Peters, ${ }^{a}$ Matthew A. P. Turner, ${ }^{\text {ab }}$ Guy J. Clarkson, ${ }^{a}$ \\ Michael D. Horbury*a and Vasilios G. Stavros (D) *a
}

\begin{abstract}
Photoisomerisation has been shown to be an efficient excited-state relaxation mechanism for a variety of nature-based and artificial-based molecular systems. Here we report on the excited-state relaxation dynamics and consequent photostability of a symmetrically functionalised cinnamate by transient electronic absorption spectroscopy, along with complementary computational and steady-state spectroscopy methods. The findings are then discussed in comparison to 2-ethylhexyl-E-4-methoxycinnamate, a structurally related 'off the shelf' chemical filter present in commercial sunscreens with a similar absorption profile. The present study allows for a like-for-like comparison beween 2-ethylhexyl-E-4methoxycinnamate and the functionalised cinnamate, driven by the need to enhance solar protection across both the UVA and UVB regions of the electromagnetic spectrum.
\end{abstract}

\section{Introduction}

Overexposure to ultraviolet radiation (UVR) is an increasing concern in today's Society. ${ }^{1-5}$ Chemical filters, found in commercial formulations, have been designed to allay such overexposure. ${ }^{6}$ Chemical filters based on cinnamates ${ }^{7,8}$ are currently being used as a class of UVR absorbing molecules in commercial formulations due to their strong absorption of UVR and potent antioxidant properties. ${ }^{9-12}$ Investigations into their photochemistry has shown a photoisomerisation pathway as a main route of deactivation, irrespective of solvent environment, ${ }^{13-17}$ and starting geometric isomer (either $E$ or $Z){ }^{14,18}$ although a change in lifetimes is noted. One such cinnamate-derivative contained in sunscreen formulations is the FDA and EU approved 2-ethylhexyl-E-4-methoxycinnamate (E-EHMC). ${ }^{6,19}$ Studies have shown that, following UVR excitation to the first electronic excited $\left(\mathrm{S}_{1}\right)$ state, E-EHMC undergoes ultrafast relaxation, repopulating the electronic ground $\left(\mathrm{S}_{0}\right)$ state through an energetically accessible $\mathrm{S}_{1} / \mathrm{S}_{0}$ conical intersection, with a percentage forming $Z$-EHMC. ${ }^{20-23}$ The formation of $Z$-EHMC is cause for concern as recent studies suggest its adverse effects on human liver cells. ${ }^{24}$ These reports

\footnotetext{
${ }^{a}$ Department of Chemistry, University of Warwick, Gibbet Hill Road, Coventry, CV4 7AL, UK. E-mail: v.stavros@warwick.ac.uk,m.horbury@warwick.ac.uk

${ }^{b}$ Department of Physics, University of Warwick, Gibbet Hill Road, Coventry, CV4 7AL, UK

$\dagger$ Electronic supplementary information (ESI) available: ${ }^{1} \mathrm{H}$ and ${ }^{13} \mathrm{C}$ NMR spectra, crystallographic data, computed structures, additional transient absorption, and steady-state spectra, fitting residuals, fluorescence quantum yields and power dependency measurements. See DOI: 10.1039/c8cp06536e
}

follow a growing trend of chemical filters with harmful photoproducts, both to humans and the environment, triggered by photoexcitation. ${ }^{24-27}$ As such, there is mounting impetus to develop 'safer' chemical filters for use in formulations, such as derivatives of commercial chemical filters.

The present work takes our current knowledge on photoisomerisation in these cinnamate systems one step further. It assesses the influence of symmetric functionalisation of cinnamates through the addition of a second methyl acrylate moiety onto the base cinnamate, methyl cinnamate (highlighted in red, Fig. 1a). This gives rise to (potentially) three distinct geometric isomers: dimethyl 3,3'-(1,4-phenylene) $\left(2 E, 2^{\prime} E\right)$-diacrylate, dimethyl $3,3^{\prime}$-(1,4-phenylene) $\left(2 Z, 2^{\prime} E\right)$-diacrylate and dimethyl
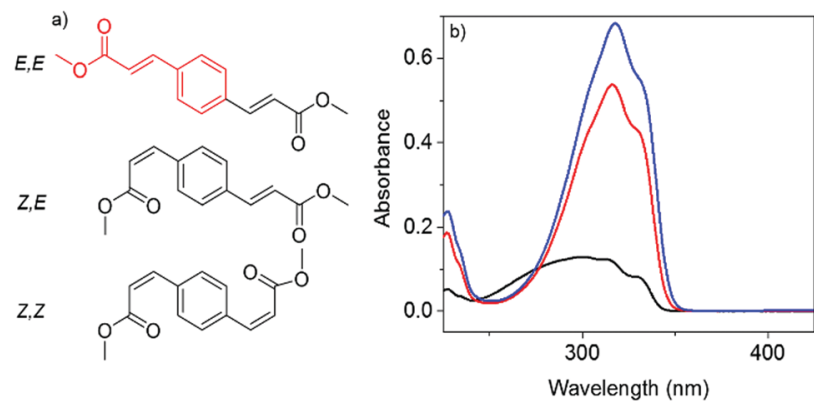

Fig. 1 (a) The three geometric isomers for DPD, with the methyl cinnamate base unit highlighted in red. (b) Steady-state absorption spectra for E,E-DPD: at $\sim 10 \mu \mathrm{M}$ concentration in ethanol (blue line) and acetonitrile (red line), and (owing to poorer solubility) $<10 \mu \mathrm{M}$ for E,E-DPD in cyclohexane (black). 
3,3'-(1,4-phenylene)(2Z,2'Z)-diacrylate, henceforth termed $E, E$ DPD, $Z, E$-DPD and $Z, Z$-DPD respectively and all shown in Fig. 1a. Drawing inspiration from the recent gas-phase experiments reported by Kinoshita et al. on the influence of hydroxy substitution on the photochemistry of methyl hydroxycinnamates ${ }^{28}$ we study the photochemistry of the geometric isomer $E, E$-DPD and track its possible evolution into the remaining geometric isomers (vide supra). In doing so we attempt to grasp a handle, on the influence of multiple geometric structures on dynamics, and how these may ultimately influence function.

To facilitate these studies, we use a combination of synthesis, spectroscopy and theory. $E, E$-DPD was synthesised following a literature procedure. ${ }^{29}$ We subsequently used femtosecond transient electronic (UV/visible) absorption spectroscopy (TEAS) to probe $(E, E$-DPD) in three solvents of varying polarity and ability to hydrogen bond: cyclohexane, acetonitrile and ethanol. Complementary time dependent density functional theory (TD-DFT) was also carried out to elucidate the relative energies of the different geometric isomers and provide insight into their associated electronic structure, ultimately assisting in the assignment of the relaxation dynamics of these systems.

\section{Experimental}

\section{Synthesis}

DPD was prepared using a method adapted from the literature. ${ }^{29}$ Briefly, 5 drops of dimethylformamide $(99.8 \%$ purity, Sigma-Aldrich) were added to a mixture of $1 \mathrm{~g} p$-phenylenediacrylic acid (97\% purity, Sigma-Aldrich) and $10 \mathrm{ml}$ thionyl chloride ( $97 \%$ purity, Sigma-Aldrich) and was refluxed overnight. The mixture was allowed to cool and the excess of thionyl chloride was removed at reduced pressure to give a yellow powder. $50 \mathrm{ml}$ of methanol (99\% purity, Alfa-Aesar) was added gradually to the powder, which started to reflux due to the exothermic nature of the reaction. The mixture was refluxed for 4 hours and then the solid was isolated via filtration. The reaction mixture was analysed using thin layer chromatography (TLC) with dichloromethane (DCM, 99.8\% purity, SigmaAldrich) as an eluent, which showed that the starting material had been consumed and that multiple new spots were visualised under UV irradiation. The mixture was recrystallized using a 2:1 solution of DCM and petrol (Sigma-Aldrich), to form a light-yellow crystalline product. The resulting compound was analysed using ${ }^{1} \mathrm{H},{ }^{13} \mathrm{C}$ NMR along with X-ray crystallography (see ESI, † Fig. S1-S4).

\section{Transient electronic absorption spectroscopy (TEAS)}

TEAS measurements were executed within the Warwick Centre for Ultrafast Spectroscopy (WCUS; www.go.warwick.ac.uk/WCUS), the capabilities of which have been previously detailed and thus only summarised here. ${ }^{30} 800 \mathrm{~nm}$ pulses $(12 \mathrm{~W}, 1 \mathrm{kHz}, 40 \mathrm{fs})$ were generated by a commercially available Ti-Sapphire regenerative amplified laser system (Spectra-Physics, Dual Ascend Pumped Spitfire Ace) seeded by a Mai Tai (Spectra-Physics). The beam was split into four fractions, each having an independent recompression grating. One of these beams $(3.5 \mathrm{~W})$ was used to produce the pump and probe pulses. The pump beam $(2.5 \mathrm{~W})$ seeds an optical parametric amplifier (Topas-Prime with UV extension, Light Conversion) allowing variability in pump wavelength. The wavelengths of excitation were: cyclohexane $315 \mathrm{~nm}$, ethanol $318 \mathrm{~nm}$, and acetonitrile $317 \mathrm{~nm}$, corresponding to the peak maxima of $E, E$-DPD for each solvent as shown in Fig. 1. The pump beam was focussed beyond the sample holder, to give a beam diameter of $500 \mu \mathrm{m}$ at the sample. $5 \%$ of the remaining $1 \mathrm{~W}$ of fundamental $800 \mathrm{~nm}$ was further attenuated and irised $(<0.05 \mathrm{~W})$, before being focussed into a vertically translated $2 \mathrm{~mm}$ thick $\mathrm{CaF}_{2}$ window to generate a white light supercontinuum from 330 to $680 \mathrm{~nm}$. The relative polarisation between the pump and probe pulses was held at magic angle $\left(54.7^{\circ}\right)$ to negate dynamics from ensemble molecular reorientation. Pump-probe time-delays $(\Delta t)$ were achieved out to the maximum temporal delay of $2.5 \mathrm{~ns}$ using a motorized optical delay line in the probe beam. Changes in optical density $(\Delta \mathrm{OD})$ were measured through the change in probe intensities using a fibre-coupled spectrometer (Avantes, AvaSpec-ULS1650F). Collated spectra were subsequently chirp corrected using the KOALA package. ${ }^{31}$ Samples were prepared in $1 \mathrm{mM}$ concentrations and delivered to the interaction region through a demountable liquid cell (Harrick Scientific Products Inc.) with a sample thickness of $100 \mu \mathrm{m}$ placed between two $\mathrm{CaF}_{2}$ windows. The sample was flowed through the cell by a diaphragm pump (SIMDOS 02) at a rate to ensure that fresh sample was interrogated with each laser pulse.

\section{Steady-state spectroscopy}

Steady-state UV/Vis absorption measurements were taken on a Cary 60 spectrophotometer with sample concentrations of $\sim 10 \mu \mathrm{M}$. Steady-state irradiation (of varying time-delay) measurements of $\sim 10 \mu \mathrm{M}$ samples (ethanol and acetonitrile solvents, $>10 \mu \mathrm{M}$ in cyclohexane) were also taken using a Horiba FluroLog-3, with an $8 \mathrm{~nm}$ slit width centred on the TEAS excitation wavelength and irradiation power equal to that of solar irradiance. Samples (each $10 \mathrm{mM},>10 \mathrm{mM}$ in cyclohexane) for ${ }^{1} \mathrm{H}$ NMR analysis, were irradiated for $1 \mathrm{~h}$ using the full available power of the Xenon lamp at the TEAS excitation wavelength using an $8 \mathrm{~nm}$ slit width. Samples were then dried of solvent and subsequently dissolved in deuterated chloroform for ${ }^{1} \mathrm{H}$ NMR analysis. Fluorescence measurements were performed on the FluroLog-3, at excitation wavelengths matching those for the TEAS measurements. A $2.5 \mathrm{~nm}$ slit width was applied for both excitation and emission measurements. A quartz cuvette with a $1 \mathrm{~cm}$ path length was used for UV/Vis, fluorescence, steady-state irradiation and ${ }^{1} \mathrm{H}$ NMR irradiation measurements.

\section{Computational}

The structures of DPD were generated for all three isomers $(E, E, E, Z, Z, Z)$, using visual molecular dynamics, ${ }^{32}$ with the molefacture plugin. Each of these structures underwent a geometry optimization within density functional theory using a cc-pVTZ basis set and PBE0 functional, ${ }^{33,34}$ using the 
NWChem software. ${ }^{35}$ Following this, time-dependant density functional theory calculations were conducted on the optimized geometries of the $E, E, E, Z$ and $Z, Z$ forms. Again, the level of theory was cc-pVTZ/PBE0 and the NWChem software was employed, generating the vertical excitation energies along with the oscillator strengths for each transition. For all calculations, a conductorlike screening model (COSMO) was used to replicate the effect of the solvent. ${ }^{36,37}$ The default COSMO implicit solvent model for each environment within NWChem was used, the descriptors of which are based on the Minnesota Solvent Descriptor Database. ${ }^{38}$ Computational output files, for the discussed calculations are available at: DOI: 10.5281/zenodo.1305150.

\section{Results}

The dominant product of the synthesis was found to be $E, E$-DPD, determined through X-ray crystallography, which showed half the molecule in the asymmetric unit with an inversion centre in the middle of the phenyl ring, and $E$ stereochemistry at each double bond. ${ }^{39}{ }^{1} \mathrm{H}$ NMR spectra also confirms the $E, E$-isomer as the major reaction product (see ESI, $\dagger$ Fig. S1). Computed structures predict that the lowest energy structure is the $E, E$-isomer, showing good agreement with the experimental structures (see ESI, $\dagger$ Fig. S5). Table 1 shows the calculated energy difference between the three isomers,

Table 1 Energies of the three possible geometric isomers of DPD, relative to the energy of $E, E-D P D$, along with first singlet (vertical) excitation energies, in acetonitrile, ethanol and cyclohexane

\begin{tabular}{lllll}
\hline Solvent & Isomer & $E, E$ & $E, Z$ & $Z, Z$ \\
\hline Acetonitrile & $\begin{array}{l}\text { Ground state difference } \\
\text { relative to } E, E \text {-DPD (eV) }\end{array}$ & & 0.27 & 0.54 \\
& $\begin{array}{l}\text { Vertical excitation } \\
\text { energies (eV, nm) }\end{array}$ & $3.79,327$ & $3.80,326$ & $3.81,325$ \\
Ethanol & $\begin{array}{l}\text { Ground state difference } \\
\text { relative to } E, E \text {-DPD (eV) }\end{array}$ & & 0.27 & 0.55 \\
& $\begin{array}{l}\text { Vertical excitation } \\
\text { energies (eV, nm) }\end{array}$ & $3.76,330$ & $3.80,326$ & $3.81,325$ \\
Cyclohexane & $\begin{array}{l}\text { Ground state difference } \\
\text { relative to } E, E \text {-DPD (eV) } \\
\text { Vertical excitation } \\
\text { energies (eV, nm) }\end{array}$ & $3.80,326$ & $3.81,325$ & $3.81,325$ \\
& & & &
\end{tabular}

along with the first singlet vertical excitations of each isomer in each solvent.

Fig. 2 shows the transient absorption spectra (TAS) resulting from photoexcitation of $E, E$-DPD, at the absorption maximum: $317 \mathrm{~nm}(3.91 \mathrm{eV})$ in acetonitrile and $318 \mathrm{~nm}(3.90 \mathrm{eV})$ in ethanol (cf. Fig. 1). We note the good agreement between the calculated vertical and experimentally measured excitation energies for E,E-DPD with differences being between 10-12 nm across all solvents. From the TAS presented as false colour heat maps in Fig. $2 \mathrm{a}$ and b, two main features are clearly visible: a stimulated emission centred at $375 \mathrm{~nm}$ and a strong excited state absorption centred at $525 \mathrm{~nm}$. We add here that in Fig. $2 \mathrm{~b}$, we see evidence of a doublet around $350 \mathrm{~nm}$, due to the ground state bleach and stimulated emission being spectrally offset in ethanol ( $c f$. acetonitrile this is a single feature). This is also evidenced in the evolutionary associated difference spectra (EADS) presented in Fig. $2 \mathrm{c}$ and $\mathrm{d}$ (discussed below). The excited state absorption and stimulated emission features return to baseline within $50 \mathrm{ps}$ leaving a small excited state absorption (not immediately apparent, highlighted in Fig. 2e and f) centred around $410 \mathrm{~nm}$, which persists to the maximum temporal delay in our measurements ( $\Delta t=2.5 \mathrm{~ns}$ ). Fig. 2e and $\mathrm{f}$ also show the presence of a ground state bleach centred at $340 \mathrm{~nm}$. We discuss this, as well as the aforementioned excited state absorption, below. We note that further studies were carried out in cyclohexane and additional excitation wavelengths, showing similar spectral features. This data is presented in the ESI, $\dagger$ Fig. S7-S9.

To quantitatively assign lifetimes to the reported TAS features, a global fit across all wavelengths (330-680 nm), using a sequential model $\left(\mathrm{A} \stackrel{\tau_{1}}{\longrightarrow} \mathrm{B} \stackrel{\tau_{2}}{\longrightarrow} \mathrm{C} \stackrel{\tau_{3}}{\longrightarrow} \mathrm{D}\right)$, was performed using the software package: Glotaran. ${ }^{40,41}$ The TAS were fitted with a three-step sequential model, with three lifetimes returned; these incorporate a convolution with a Gaussian to model our instrument response (FWHM $=80 \mathrm{fs})$. The extracted lifetimes are shown in Table 2, along with the associated errors. Transient slices are also provided in the ESI, $\dagger$ (Fig. S10 and S11) to illustrate the quality of the fit using this model. We note that where the error returned was less than $50 \%$ of our instrument response, we elected to quote an error of 40 fs (i.e. $50 \%$ of our instrument response). The corresponding EADS are also shown
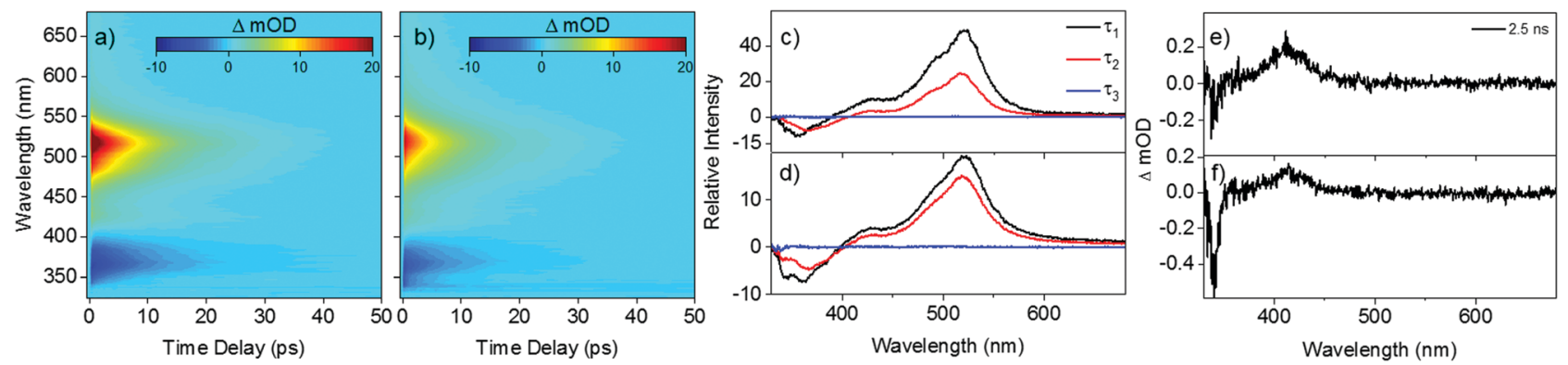

Fig. 2 False colour heat maps of E,E-DPD following photoexcitation at (a) $317 \mathrm{~nm}$ in acetonitrile and (b) $318 \mathrm{~nm}$ in ethanol. Evolutionary associated difference spectra (EADS) for the TAS of E,E-DPD in (c) acetonitrile and (d) ethanol. TAS at $\Delta t=2.5 \mathrm{~ns}$ of $E, E$-DPD in (e) acetonitrile and (f) ethanol. Note the intensity scale in (a) is cropped as a visual aid to the dynamical features beyond the first $200 \mathrm{fs}$. An expanded intensity scale of (a) is presented in the ESI, † Fig. S6. 
Table 2 Lifetimes and associated errors $(2 \sigma)$ extracted from the global sequential fitting of E,E-DPD and previously reported values $E$-EHMC, in methanol and cyclohexane

\begin{tabular}{lrrr}
\hline Solvent & \multicolumn{1}{c}{$\tau_{1}(\mathrm{fs})$} & \multicolumn{1}{c}{$\tau_{2}(\mathrm{ps})$} & $\tau_{3}(\mathrm{~ns})$ \\
\hline$E, E$-DPD & & & \\
Acetonitrile & $90 \pm 40$ & $7.73 \pm 0.04$ & $\gg 2.5$ \\
Ethanol & $400 \pm 40$ & $8.28 \pm 0.04$ & $\gg 2.5$ \\
Cyclohexane & $480 \pm 40$ & $10.34 \pm 0.15$ & $\gg 2.5$ \\
& & & \\
E-EHMC & & & \\
Methanol & $260 \pm 90$ & $1.10 \pm 0.30$ & $\gg 2.0$ \\
Cyclohexane & $600 \pm 100$ & $1.80 \pm 1.20$ & \\
${ }^{a}$ Taken from ref. 20. & & & \\
\hline
\end{tabular}

in Fig. 2c (acetonitrile) and d (ethanol). The associated residuals for each TAS (difference between experimentally measured and calculated TAS) are provided in the ESI, $\dagger$ (Fig. S12 and S13).

\section{Discussion}

From the reported lifetimes given in Table 2 and associated EADS presented in Fig. 2c and d, we can now discuss the reported TAS, with reference to the schematic shown in Fig. 3. We note that we draw considerable insight from comparative studies (with associated lifetimes; also shown in Table 2) of E-EHMC. ${ }^{20}$ Photoexcitation to the $\mathrm{S}_{1}\left({ }^{1} \pi \pi^{*}\right)$ state leads to population traversing out of the Franck-Condon region described by $\tau_{1}$. Notably $\tau_{1}$ for acetonitrile is significantly shorter than that of either ethanol or cyclohexane, as has previously been reported for similar systems. ${ }^{42,43}$ This also (likely) encompasses any geometry rearrangement of solute and surrounding solvent. As with similar systems, this geometry rearrangement is not thought to involve a change in the electronic state. ${ }^{16,44} \tau_{2}$ then describes the evolution of excited-state population, as it moves towards an $\mathrm{S}_{1} / \mathrm{S}_{0}$ conical intersection along the $E \rightarrow Z$ isomerisation coordinate and then funnels through this conical intersection. This latter step leads to effective repopulation of the ground $\left(\mathrm{S}_{0}\right)$ state. Previous reports of photoisomerisation,,$^{7,15,18,20,42,43}$ have

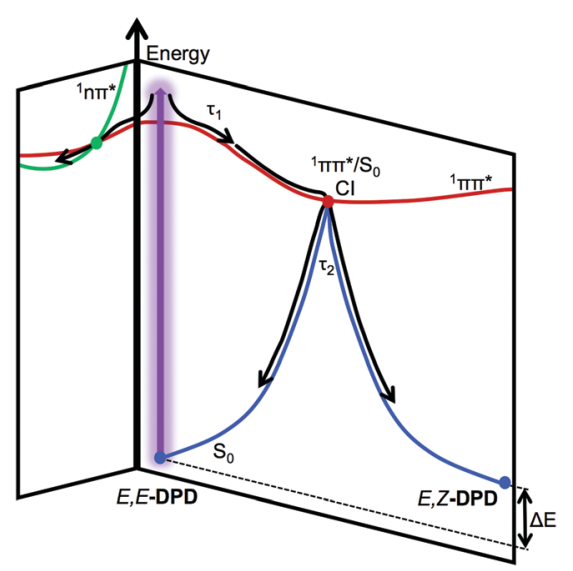

Fig. 3 Schematic of relaxation for photoexcited E,E-DPD, showing the progression of $\tau_{1}$ and $\tau_{2}$. We add that $\tau_{3}$ (not shown) would correspond to population trapped in the E,Z-DPD and ${ }^{1} \mathrm{n} \pi^{\star}$ state. identified the presence of an absorption feature at long time delays, attributed to the formation of the corresponding geometric isomer; in the present case, this would correspond to $E, Z$-DPD (or $Z, Z$-DPD). This may be the case here, evidenced through the incomplete ground state bleach recovery of $E, E$-DPD (negative feature $<350 \mathrm{~nm}$; see Fig. 2e and f). However, the positive feature extending $>400 \mathrm{~nm}$ points towards a long-lived excited state, which would also restrict ground state bleach recovery. We attribute this long-lived excited state to either an ${ }^{1} \mathrm{n} \pi^{*}$ or triplet state. We draw confidence that this is due to an excited state absorption, given that neither E,Z-DPD or Z,Z-DPD isomer is likely to absorb beyond $400 \mathrm{~nm}$ (see Fig. 4). Both these features in the TAS, persist to $\Delta t=2.5 \mathrm{~ns}$ and are assigned a
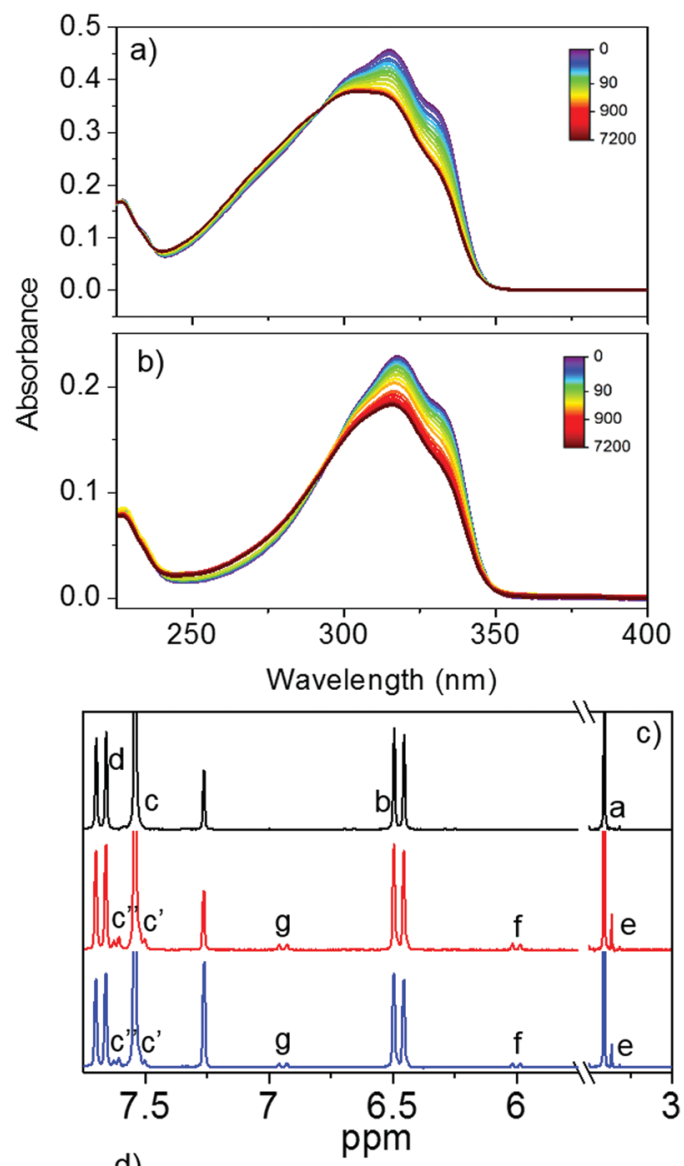

d)

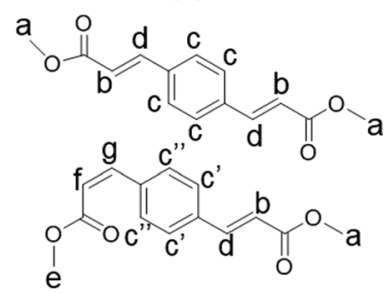

Fig. 4 Steady-state UV/Vis absorption of $10 \mu M E, E-D P D$ in (a) acetonitrile and (b) ethanol, following irradiation at solar fluences over the peak absorption $\left(\lambda_{\max }=317 \mathrm{~nm}\right.$ (acetonitrile) and $318 \mathrm{~nm}$ (ethanol)). The colour bar indicates length of irradiation in seconds. Selected region of (c) pre- (black) and post-irradiation ${ }^{1} \mathrm{H}$ NMR of E,E-DPD irradiated at $10 \mathrm{mM}$ concentration in acetonitrile and ethanol (red and blue respectively). (d) Peak assignment of chemical shifts in $E, E$ and $E, Z$ isomers. 
lifetime of $\tau_{3}$. We discuss these features and the origin of $\tau_{3}$ in further detail below.

Steady-state irradiation at solar irradiance intensity was conducted to explore the long term photostability of $E, E$-DPD upon exposure to UVR. The results for both acetonitrile and ethanol are shown in Fig. 4; steady-state irradiation studies for $E, E$-DPD in cyclohexane are shown in the ESI. $\dagger$ All absorption spectra show a depletion of the initial main peak before plateauing following 20 minutes (1200 seconds) of irradiation. Accompanying this depletion is a small shift in the absorption to higher energy. The depletion of absorbance, is assigned to the generation of a photoequilibrium between $E, E$-DPD and E,Z-DPD; see Fig. 1 for associated structures. This depletion is consistent with the calculated oscillator strengths $\left(\mathrm{S}_{1} \leftarrow \mathrm{S}_{0}\right.$, at the calculated absorption maximum) between the two geometric isomers; e.g. 1.30 and 1.17 (arb. units) for $E, E$-DPD and $E, Z$-DPD respectively in acetonitrile. To further prove the existence of this photoequilibrium, and to exclude formation of the $Z, Z$-isomer, ${ }^{1} \mathrm{H}$ NMR of the irradiated samples was performed. The samples were irradiated for $5 \mathrm{~h}$ using the full power of the Xenon lamp (approximately $30 \mathrm{~mW})$ at the absorption maximum $\left(\lambda_{\max }=317\right.$ and $318 \mathrm{~nm}$, for acetonitrile and ethanol respectively), to obtain sufficient conversion to the E,Z-DPD for ${ }^{1} \mathrm{H}$ NMR identification; the sample concentration was also increased to $10 \mathrm{mM}$ to facilitate this. The ${ }^{1} \mathrm{H}$ NMR spectra in deuterated chloroform for the region of interest for both pre- and post-irradiation are shown in Fig. 4c.

From the analysis of the ${ }^{1} \mathrm{H}$ NMR spectra, we can conclude that $E \rightarrow Z$ isomerisation occurs, with peaks $\mathrm{f}$ and $\mathrm{g}$ showing a $13 \mathrm{~Hz}$ splitting characteristic of the $Z$-isomer, along with the appearance of peak e indicating a change in environment of the terminating methyl. This is in contrast to peak $\mathrm{b}$ and $\mathrm{d}$ which have a splitting of $16 \mathrm{~Hz}$ (see ESI, $\dagger$ Fig. S3), attributed to $E$-isomer. One would anticipate a progression towards $Z, Z$-DPD to be a stepwise multiple-photon process, with the first conversion to $E, Z$-DPD then subsequently conversion to $Z, Z$-DPD. From the NMR we can deduce that no detectable presence of $Z, Z$-DPD is observed, confirmed through the splitting of the hydrogens on the aromatic ring (see signals $\mathrm{c}^{\prime}$ and $\mathrm{c}^{\prime \prime}$ ) indicating unsymmetrical substitution.

To conclude our discussion, we shall return to the nature of the long-lived features in the TAS (Fig. 2e and f) and the origin of $\tau_{3}$. It is evident from steady-state irradiation measurements that the geometric isomer E,Z-DPD has a weaker absorbance $<350 \mathrm{~nm}$ than the $E, E$-DPD ( $c f$. Fig. $4 \mathrm{a}$ and b, 0 and 7200 seconds irradiation). Given the TAS represents a difference spectrum (post-irradiated sample compared to pre-irradiated sample), it is unsurprising that we see a negative feature in the TAS (along with an incomplete, and overlapping, ground state bleach recovery) and is further evidenced, via ${ }^{1} \mathrm{H}$ NMR data, of isomerisation from $E, E$-DPD $\rightarrow E$,Z-DPD. However, there is no absorption of either isomer at wavelengths greater than $350 \mathrm{~nm}$, pointing to a different origin of the positive feature in the TAS $>400 \mathrm{~nm}$. This relatively small excited state absorption is likely due to either a ${ }^{1} n \pi^{*}$ or a triplet state. Several reports have suggested the presence of ${ }^{1} n \pi^{*}$ states playing a role within relaxation of similar systems. ${ }^{8,28,45}$
Fluorescence has also been reported for similar systems ${ }^{7,20,46}$ and was again observed here with the quantum yield reported as $0.039 \pm 0.002$ in cyclohexane (see ESI, $\dagger$ Fig. S17), hence a relatively minor pathway. Also, no phosphorescence was observed. The possibility of the absorption feature being due to a radical cation species, generated via a step-wise two-photon ionisation, was considered (as seen for similar species), ${ }^{9,17,42}$ but dismissed due to the linear power dependence of the feature (see ESI, $\dagger$ Fig. S19). Taken together, we assign $\tau_{3}$ to a combination of a longlived ${ }^{1} \mathrm{n} \pi^{*}$ state and presence of E,Z-DPD. We conclude our discussion of the long lived feature in the TAS by noting that a quantitative analysis of the conversion of $E, E$-DPD to $E, Z$-DPD is not possible from the TAS given the convoluted nature of the features both at early times (from the stimulated emission) and at $\Delta t=2.5 \mathrm{~ns}$ (from the presence of the ${ }^{1} \mathrm{n} \pi^{*}$ state).

It is evident from the data thus far that $E, E$-DPD may possess suitable properties for applications as a chemical filter. Notably, its absorption spectrum is similar to that of $E$-EHMC, ${ }^{19,20}$ but with a slight red-shift $(\sim 10 \mathrm{~nm})$, meaning it extends further into the UVA which is a sought-after quality for a chemical filter. Coupled to this is the rapid relaxation of the excited state through an accessible conical intersection, consistent with the previous literature of similar species. ${ }^{7,42,43}$ Furthermore, the apparent degradation upon constant UVR is attributed to interconversion to the $E, Z$-DPD isomer. This finding is, once again, in good agreement with previous reports on E-EHMC versus $Z$-EHMC. ${ }^{20,23}$

Whilst there are similarities to $E$-EHMC, there are evident differences. Firstly, the apparent interconversion between the two isomers is longer for $E$,E-DPD in comparison with $E$-EHMC; e.g. in cyclohexane, $\tau_{2} \sim 10 \mathrm{ps}$ for $E$,E-DPD versus $\sim 2 \mathrm{ps}$ for $E$-EHMC (see Table 2). ${ }^{20}$ This indicates that photoisomerisation is impinged by the different substituent in the para position. In this case, the addition of a second methyl acrylate alters the excited state dynamics by increasing the time for ground state repopulation (be it in $E, Z$-DPD (more likely) or $E, E$-DPD (less likely), Fig. 3). Whilst the underlying reason for this is not known, this inevitably points to alterations to the excited state landscape along the photoisomerisation coordinate. We add here that the increase in excited state lifetime could promote competing pathways; the ns component $\left(\tau_{3}\right)$ of the $E$-EHMC can entirely be attributed to occurrence of the $Z$-isomer whilst for $E, E$-DPD, this appears to be a multicomponent, with the front runners being the $E, Z$-DPD isomer and a long-lived $n \pi^{*}$ state. Additionally, compared to $E$-EHMC, the time taken to reach a photoequilibrium is increased to 20 minutes ( $c f .2$ minutes for $E$-EHMC). ${ }^{23}$ We finally note that no detectable spectroscopic evidence for $Z, Z$-DPD was observed in any experiments, leading to the hypothesis that a photoequilibrium is only established between $E, E$-DPD and $E, Z$-DPD.

\section{Conclusions}

This study highlights the effect of chemical substitution on an established photoisomerisation pathway. In this instance, we compare $E, E$-DPD with the commercial chemical filter $E$-EHMC. 
Following photoexcitation in solvents of varying polarity, the excited state population in E,E-DPD evolves out of the FranckCondon region within $500 \mathrm{fs}$, along with geometry rearrangement of the solute and surrounding solvent, enabling access to an energetically accessible $S_{1} / S_{0}$ conical intersection. As the population transfers to the electronic ground state, the first difference between $E, E$-DPD and $E$-EHMC is in the relaxation times; $\sim 10$ ps for $E$,E-DPD and $\sim 2$ ps $E$-EHMC (both in cyclohexane). Ultimately this change is assigned to effects of the additional methyl acrylate on the excited state landscape. The second difference relates to the assignment of $\tau_{3}$. Here $\tau_{3}$ describes the presence of the corresponding $E, Z$-DPD isomer, along with a small percentage of the excited state population trapped in an ${ }^{1} \mathrm{n} \pi^{*}$ (or triplet) state, which persists beyond the time-window of the present measurements ( $<\mathrm{ns}$ ). This ns component is absent in $E$-EHMC. Furthermore no evidence for Z,Z-DPD was observed, leading to the conclusion that its formation is unfavoured and the photoequilibrium is generated between $E, E$-DPD, and $E, Z$-DPD.

As a final comparison, the steady-state absorption spectra of $E, E$-DPD (in solvents of varying polarity) are red-shifted relative to the absorption spectra of $E$-EHMC, allowing for stronger absorption within the UV-A region of the spectrum. This is a key quality, which is expected for new chemical filters, given the growing need for photoprotection across the UVB and UVA regions of the solar spectrum. ${ }^{1}$ Taken together the results shown here provide a potential blue-print into how a current chemical filter can be adapted and improved for use in future cosmetic products.

\section{Conflicts of interest}

There are no conflicts to declare.

\section{Acknowledgements}

The authors thank Dr Michael Staniforth at the Warwick Centre for Ultrafast Spectroscopy (WCUS; www.go.warwick.ac.uk/WCUS), for experimental aid. The authors would also like to thank Dr Nicholas Hine for valuable discussions regarding the theory. J. M. W. is grateful to EPSRC and Newport Spectra-Physics Ltd for a joint studentship. M. A. P. T. thanks EPSRC for a doctoral studentship through the EPSRC Centre for Doctoral Training in Molecular Analytical Science, grant number EP/L015307/1. M. D. H. thanks the Leverhulme Trust for postdoctoral funding. V. G. S. is grateful to the EPSRC for an equipment grant (EP/N010825) and the Royal Society and the Leverhulme Trust for a Royal Society Leverhulme Trust Senior Research Fellowship. Computing facilities were provided by the Scientific Computing Research Technology Platform of the University of Warwick.

\section{Notes and references}

1 R. P. Sinha and D.-P. Häder, Photochem. Photobiol. Sci., 2002, 1, 225-236.

2 G. P. Pfeifer, Y. H. You and A. Besaratinia, Mutat. Res., Fundam. Mol. Mech. Mutagen., 2005, 571, 19-31.
3 F. R. de Gruijl, Eur. J. Cancer, 1999, 35, 2003-2009.

4 M. Brenner and V. J. Hearing, Photochem. Photobiol., 2008, 84, 539-549.

5 M. Krause, A. Klit, M. Blomberg Jensen, T. Søeborg, H. Frederiksen, M. Schlumpf, W. Lichtensteiger, N. E. Skakkebaek and K. T. Drzewiecki, Int. J. Androl., 2012, 35, 424-436.

6 N. A. Shaath, Photochem. Photobiol. Sci., 2010, 9, 464-469.

7 M. D. Horbury, L. A. Baker, N. D. N. Rodrigues, W.-D. Quan and V. G. Stavros, Chem. Phys. Lett., 2017, 673, 62-67.

8 N. D. N. Rodrigues, M. Staniforth, J. D. Young, Y. Peperstraete, N. C. Cole-Filipiak, J. R. Gord, P. S. Walsh, D. M. Hewett, T. S. Zwier and V. G. Stavros, Faraday Discuss., 2016, 194, 709-729.

9 S. Foley, S. Navaratnam, D. J. McGarvey, E. J. Land, T. G. Truscott and C. A. Rice-Evans, Free Radical Biol. Med., 1999, 26, 1202-1208.

10 A. Gaspar, M. Martins, P. Silva, E. M. Garrido, J. Garrido, O. Firuzi, R. Miri, L. Saso and F. Borges, J. Agric. Food Chem., 2010, 58, 11273-11280.

11 J. C. Murray, J. A. Burch, R. D. Streilein, M. A. Iannacchione, R. P. Hall and S. R. Pinnell, J. Am. Acad. Dermatol., 2008, 59, 418-425.

12 K. Sevgi, B. Tepe and C. Sarikurkcu, Food Chem. Toxicol., 2015, 77, 12-21.

13 J. Luo, Y. Liu, S. Yang, A. L. Flourat, F. Allais and K. Han, J. Phys. Chem. Lett., 2017, 8, 1025-1030.

14 S. Wang, S. Schatz, M. C. Stuhldreier, H. Böhnke, J. Wiese, C. Schröder, T. Raeker, B. Hartke, J. K. Keppler, K. Schwarz, F. Renth and F. Temps, Phys. Chem. Chem. Phys., 2017, 19, 30683-30694.

15 M. D. Horbury, W.-D. Quan, A. L. Flourat, F. Allais and V. G. Stavros, Phys. Chem. Chem. Phys., 2017, 19, 21127-21131.

16 A. Espagne, D. H. Paik, P. Changenet-Barret, M. M. Martin and A. H. Zewail, ChemPhysChem, 2006, 7, 1717-1726.

17 M. Vengris, D. S. Larsen, M. A. van der Horst, O. F. A. Larsen, K. J. Hellingwerf and R. van Grondelle, J. Phys. Chem. B, 2005, 109, 4197-4208.

18 M. D. Horbury, A. L. Flourat, S. E. Greenough, F. Allais and V. G. Stavros, Chem. Commun., 2018, 54, 936-939.

19 U. Osterwalder and B. Herzog, Photochem. Photobiol. Sci., 2010, 9, 470-481.

20 Y. Peperstraete, M. Staniforth, L. A. Baker, N. D. N. Rodrigues, N. C. Cole-Filipiak, W.-D. Quan and V. G. Stavros, Phys. Chem. Chem. Phys., 2016, 18, 28140-28149.

21 T. M. Karpkird, S. Wanichweacharungruang and B. Albinsson, Photochem. Photobiol. Sci., 2009, 8, 1455-1460.

22 S. Pattanaargson, T. Munhapol, P. Hirunsupachot and P. Luangthongaram, J. Photochem. Photobiol., A, 2004, 161, 269-274.

23 K. M. Hanson, S. Narayanan, V. M. Nichols and C. J. Bardeen, Photochem. Photobiol. Sci., 2015, 14, 1607-1616.

24 A. Sharma, K. Bányiová, P. Babica, N. El Yamani, A. R. Collins and P. Čupr, Sci. Total Environ., 2017, 593-594, 18-26.

25 N. Blüthgen, S. Zucchi and K. Fent, Toxicol. Appl. Pharmacol., 2012, 263, 184-194. 
26 M. Coronado, H. De Haro, X. Deng, M. A. Rempel, R. Lavado and D. Schlenk, Aquat. Toxicol., 2008, 90, 182-187.

27 M. Ghazipura, R. McGowan, A. Arslan and T. Hossain, Reprod. Toxicol., 2017, 73, 175-183.

28 S. Kinoshita, Y. Miyazaki, M. Sumida, Y. Onitsuka, H. Kohguchi, Y. Inokuchi, N. Akai, T. Shiraogawa, M. Ehara, K. Yamazaki, Y. Harabuchi, S. Maeda, T. Taketsugu and T. Ebata, Phys. Chem. Chem. Phys., 2018, 20, 17583-17598.

29 H. Miura, S. Takahashi, H. Kasai, S. Okada, K. Yase, H. Oikawa and H. Nakanishi, Cryst. Growth Des., 2010, 10, 510-517.

30 J. M. Woolley, M. Staniforth, M. D. Horbury, G. W. Richings, M. Wills and V. G. Stavros, J. Phys. Chem. Lett., 2018, 9, 3043-3048.

31 M. P. Grubb, A. J. Orr-Ewing and M. N. R. Ashfold, Rev. Sci. Instrum., 2014, 85, 64104.

32 W. Humphrey, A. Dalke and K. Schulten, J. Mol. Graphics, 1996, 14, 33-38.

33 T. H. Dunning, J. Chem. Phys., 1989, 90, 1007-1023.

34 C. Adamo and V. Barone, J. Chem. Phys., 1999, 110, 6158-6170.

35 M. Valiev, E. J. Bylaska, N. Govind, K. Kowalski, T. P. Straatsma, H. J. J. Van Dam, D. Wang, J. Nieplocha, E. Apra, T. L. Windus and W. A. D. Jong, Comput. Phys. Commun., 2010, 181, 1477-1489.
36 A. Klamt and G. Schüürmann, J. Chem. Soc., Perkin Trans. 2, 1993, 799-805.

37 D. M. York and M. Karplus, J. Phys. Chem. A, 1999, 103, 11060-11079.

38 P. Winget, D. M. Dolney, D. J. Giesen, C. J. Cramer and D. G. Truhlar, Minnesota solvent descriptor database, http://comp.chem.umn.edu/solvation/mnsddb.pdf.

39 K. Ueno, H. Nakanishi and M. Hasegawa, Acta Crystallogr., Sect. B: Struct. Sci., Cryst. Eng. Mater., 1978, 34, 2034-2035.

40 J. J. Snellenburg, S. P. Laptenok, R. Seger, K. M. Mullen and I. H. M. van Stokkum, J. Stat. Softw., 2012, 49, 1-22.

41 K. M. Mullen and I. H. M. van Stokkum, J. Stat. Softw., 2007, 18, 1-46.

42 M. D. Horbury, L. A. Baker, W.-D. Quan, S. E. Greenough and V. G. Stavros, Phys. Chem. Chem. Phys., 2016, 18, 17691-17697.

43 L. A. Baker, M. D. Horbury, S. E. Greenough, F. Allais, P. S. Walsh, S. Habershon and V. G. Stavros, J. Phys. Chem. Lett., 2016, 7, 56-61.

44 H. Kuramochi, S. Takeuchi and T. Tahara, J. Phys. Chem. Lett., 2012, 3, 2025-2029.

45 X. P. Chang, C. X. Li, B. Bin Xie and G. Cui, J. Phys. Chem. A, 2015, 119, 11488-11497.

46 M. Promkatkaew, S. Suramitr, T. Karpkird, S. Wanichwecharungruang, M. Ehara and S. Hannongbua, Photochem. Photobiol. Sci., 2014, 13, 583. 